\title{
A Suitable Structure to Control the System of Quad-rotor Miniature Aerial Vehicles
}

\author{
Van-Dai Bui ${ }^{1}$, Trong-Thang Nguyen ${ }^{2}$, Ngoc-Hoan Than ${ }^{3}$, Duc-Minh Nguyen ${ }^{4}$ \\ ${ }^{1,2}$ Faculty of Energy Engineering, Thuyloi University, Vietnam \\ ${ }^{3,4}$ Department of Electrical Engineering and Automation, Haiphong Private University, Vietnam
}

\begin{tabular}{l}
\hline Article Info \\
\hline Article history: \\
Received Mar 7, 2018 \\
Revised Aug 22, 2018 \\
Accepted Sep 10, 2018 \\
\hline
\end{tabular}

\section{Keyword:}

Quad-rotor

Robot control

PID control

Miniature aerial vehicles

\begin{abstract}
Miniature Aerial Vehicles with four rotors is called Quad-rotor MAV, popularly used in aspects of life such military, civilian products, processes and remote sensor, etc. In this paper, the authors present the suitable structure of control system for the Quad-rotor MAV. The first, the Six Degrees of Freedom (6 DOF) of the Quad-rotor MAV dynamic model is built. After, the control structure with the single loop is built. But in the single-loop system, only four control signals of Quad-rotor MAV can be controlled, so the Quadrotor MAV can be reached the height only and keep the stability. However, it is important to note that we have to well-known the orbit of the Quad-rotor MAV flight; the Quad-rotor MAV must fly point-to-point exactly, so the six coordinate variables must be controlled. So, the double loop control structure system is proposed to do that. Finally, the simulation results analysis and the experimental results of the real model are explored to prove the effectiveness of the proposed structure.
\end{abstract}

Copyright () 2018 Institute of Advanced Engineering and Science. All rights reserved.

\section{Corresponding Author:}

Van-Dai Bui,

Faculty of Energy Engineering,

Thuyloi University,

175 Tay Son, Dong Da, Ha Noi, Viet Nam.

Email: daibv@tlu.edu.vn

\section{INTRODUCTION}

There is a wide range of Unmanned Aerial Vehicles (UAV) technology and satellite which under development rapidly around the globe, provide for the critical services. More, the technology of MicroElectro-Mechanical is growing, used in all the life fields such as civilian/commercial spheres, the military realm, information technology, processes, etc, making the aircraft size is smaller, so the application of aircraft in the Miniature Aerial Vehicles (MAV) field is increased. So, the research on MAV is the interesting topic in the world [1],[2].

The MAV is a small unit which is driven in conditional without the person inside, flight on the aerial by using the rotor thrust to overcome gravity. So, MAV is the remote-control or an automatic unit with the ability is bearing the weight for any task. The missions of MAV are intercept, battle damage assessment, target acquisition, surveillance, weather reconnaissance, and even attack the targets directly with the highquality features.

In the previous time, the MAV is used only for the vertical takeoff and landing (VTOL) unit, so the ability to apply in practice is less. However, the advantage studies on VTOL MAV [3]-[5] prove that VTOL MAV can be landed and taken off independently, flown in the different environments, flown beside, flown back, flown forward, etc. These advantages make the application of VTOL MAV is more developed.

The new structure and layout of VTOL MAV with Quad-rotor have been proposed in [6]-[8], called Quad-rotor MAV with the flyway is special. So, the researchers are attracted to find out about Quad-rotor MAV, which has the machine structure is simpler than the conventional single-rotor. Quad-rotor MAV can 
change the lifting force by changing the speed of any four rotors, so it can fly in space more stable, can change the position and the angle to achieve the different requirements of the flight [9],[10]. Quad-rotor MAV can bear the payload with more heavily than single-rotor. Because the Quad-rotor MAV has four rotors with the symmetric structure, there is not the twisting moment which is produced by each rotor, so it is not necessary to have an anti-torque propeller [11],[12]. Because of their wonderful advantage, Quad-rotor MAV has been used for making the lower speed aircraft, flying into the barrier area, and it is suitable for a narrow space. So, Quad-rotor MAV is the important topic of study among the researchers in the world. In this paper, the authors propose a novel structure of control system for Quad-rotor MAV. Basing on the new structure, the aircraft has excellent performance, simple structure, low cost, and can be applied in practice.

\section{THE QUAD-ROTOR MOTION EQUATION}

\subsection{The quad-rotor model}

The Quad-rotor has four rotors controlling four input forces respectively. The Quad-rotor model is shown in Figure 1.

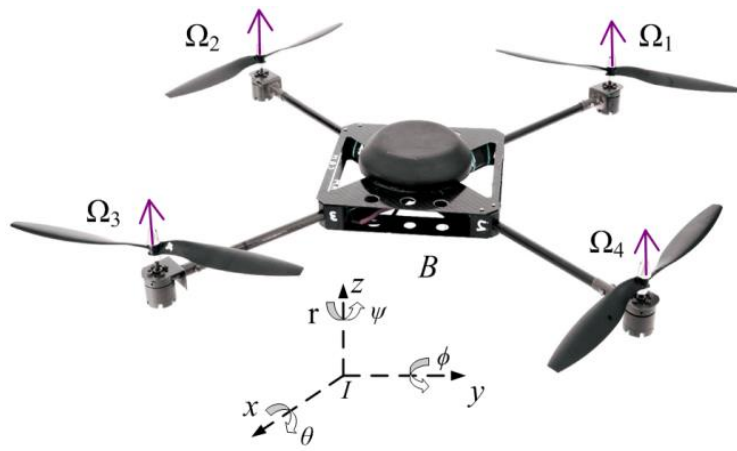

Figure 1. The Quad-rotor model and the coordinate system

The overall force is the sum of four the rotors forces. The Pitch movement is controlled by reducing the front motor speed or increasing the rear motor speed. The Roll movement is controlled by reducing the left motor speed or increasing and the right motor speed. The Yaw movement is controlled by decreasing the front motor speed or increasing the rear motor speed combined with increasing or decreasing the speed of the side motors [13]-[16].

\subsection{The Quad-rotor dynamic equations}

The Quad-rotor is a multi-variable and nonlinear object with 4 input signal controls but with 6 degrees of freedom. In order to control the coordinates of Quad-rotor from a point to the other point in the space, it is very important to know the equations of Quad-rotor dynamic model, which is shown as follows [17]-[20].

$$
\left\{\begin{array}{l}
\ddot{x}=(\sin \psi \sin \phi+\cos \psi \sin \theta \cos \phi) \frac{U_{1}}{m} \\
\ddot{y}=(-\cos \psi \sin \phi+\sin \psi \sin \theta \cos \phi) \frac{U_{1}}{m} \\
\ddot{z}=-g+(\cos \theta \cos \phi) \frac{U_{1}}{m} \\
\dot{p}=\frac{I_{y}-I_{z}}{I_{x}} q r-\frac{J_{T P}}{I_{x}} q \Omega+\frac{U_{2}}{I_{x}} \\
\dot{q}=\frac{I_{z}-I_{x}}{I_{y}} p r-\frac{J_{T P}}{I_{y}} p \Omega+\frac{U_{3}}{I_{y}} \\
\dot{r}=\frac{I_{x}-I_{y}}{I_{z}} p q+\frac{U_{4}}{I_{z}}
\end{array}\right.
$$

with $g$ is the gravitational acceleration, $\mathrm{m}$ is the weight of Quad-rotor, $J_{T P}$ is the inertia moment of the rotor. $U_{1}$, $U_{2}, U_{3}, U_{4}$ and $\Omega$ are 


$$
\left\{\begin{array}{l}
U_{1}=b\left(\Omega_{1}^{2}+\Omega_{2}^{2}+\Omega_{3}^{2}+\Omega_{4}^{2}\right) \\
U_{2}=b l\left(\Omega_{4}^{2}-\Omega_{2}^{2}\right) \\
U_{3}=b l\left(\Omega_{3}^{2}-\Omega_{1}^{2}\right) \\
U_{4}=d l\left(\Omega_{2}^{2}+\Omega_{4}^{2}-\Omega_{1}^{2}-\Omega_{3}^{2}\right) \\
\Omega=-\Omega_{1}+\Omega_{2}-\Omega_{3}+\Omega_{4}
\end{array}\right.
$$

\subsection{The equations of controller and other stages}

The PID controller has the equation in Laplace as follows:

$$
u(s)=\left(K_{p}+\frac{K_{i}}{s}+s K_{d}\right) e(s)
$$

where: $e$ is the error which is equal to the set value minus the real value. $K p$ is the gain coefficient, $K i$ is the integral coefficient, $K d$ is derivative coefficient, $u$ is the output value of the controller.

In this paper, we introduce two control structures for Quad-rotor. The first is the control structure with the single loop [21], shown in Figure 2. The second is the control structure with the double loop [22], shown in Figure 3. Both structures are included of three main parts.

The first part is the controller. In the single loop control structure, the controller is the control algorithm block. In the double loop control structure, the controller includes two parts, which are the position control block and the attitude block connected to each other. The second part is the inverse transformation block. This block is built by the equations shown as follows:

$$
\left\{\begin{array}{l}
\ddot{z}=-g+(\cos \theta \cos \phi) U_{1} / m \\
\ddot{\phi}=U_{2} / I_{x} \\
\ddot{\theta}=U_{3} / I_{y} \\
\ddot{\psi}=U_{4} / I_{z}
\end{array}\right.
$$

After the inverse transformation block, we received the signals shown as follows:

$$
\left\{\begin{array}{l}
U_{1}=(\ddot{z}+g) m /(\cos \theta \cos \phi) \\
U_{2}=I_{x} \ddot{\phi} \\
U_{3}=I_{y} \ddot{\theta} \\
U_{4}=I_{z} \ddot{\psi}
\end{array}\right.
$$

The third part is the convert block of rotor speed. The equations of this block are as follows:

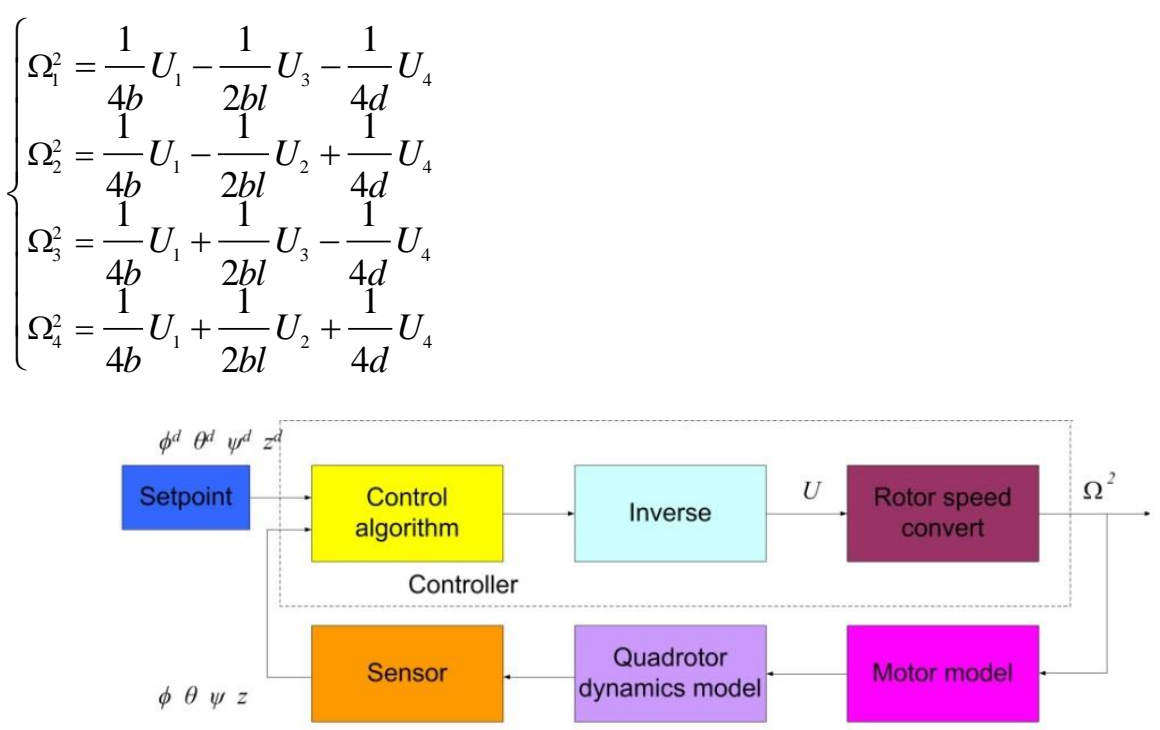

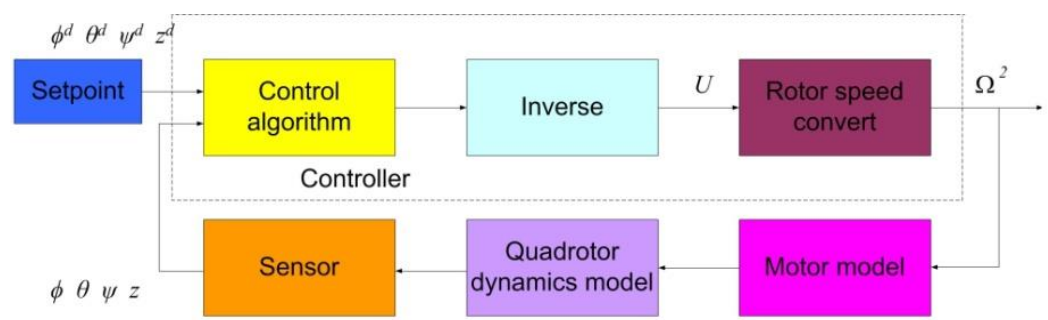


Figure 2. The diagram of the control structure with single loop

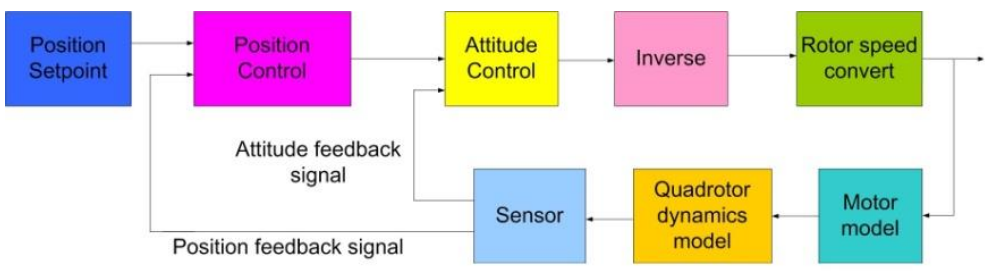

Figure 3. The diagram of the control structure with double loop

\section{BUILDING THE SIMULATION MODEL}

\subsection{The model of single loop control structure}

We make the simulation diagram on the Matlab-Simulink software. The control structure with the single loop is shown in Figure 4.

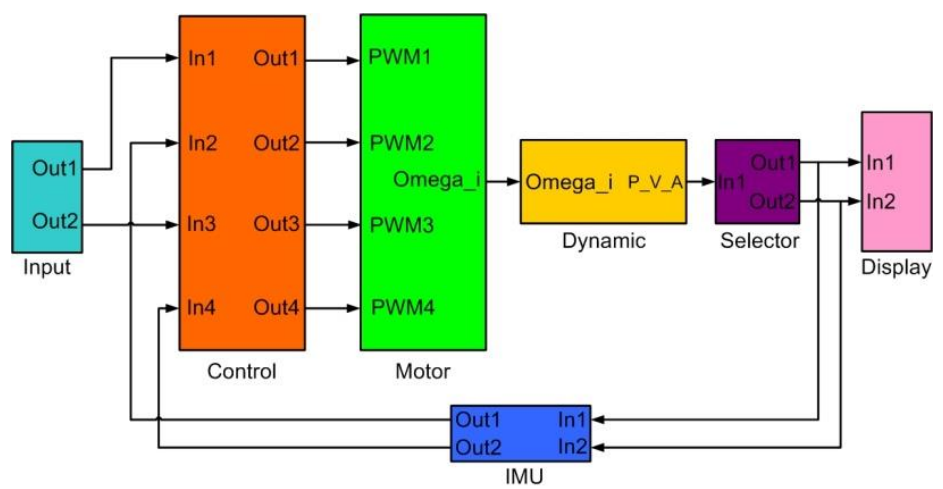

Figure 4. The single loop control structure

In Figure 4, the input block is the set values of the system. The control block is presented in detail as Figure 5. The Motor block is built according to the equation (2) with the role is converting the output value of controller into the value of motor speed $\Omega_{1}, \Omega_{2}, \Omega_{3}, \Omega_{4}$. The dynamic block is built by the equations of Quadrotor aircraft (1). The selector block is presented in detail as figure 6 , with the role is selecting the signals such as height $(z)$, roll $(\phi)$, pitch $(\theta)$ and yaw $(\psi)$ from the dynamic block into the IMU block. The IMU block is the measurement system which measures height $(\mathrm{z})$, roll $(\phi)$, pitch $(\theta)$ and yaw $(\psi)$ signals send to the controller. 


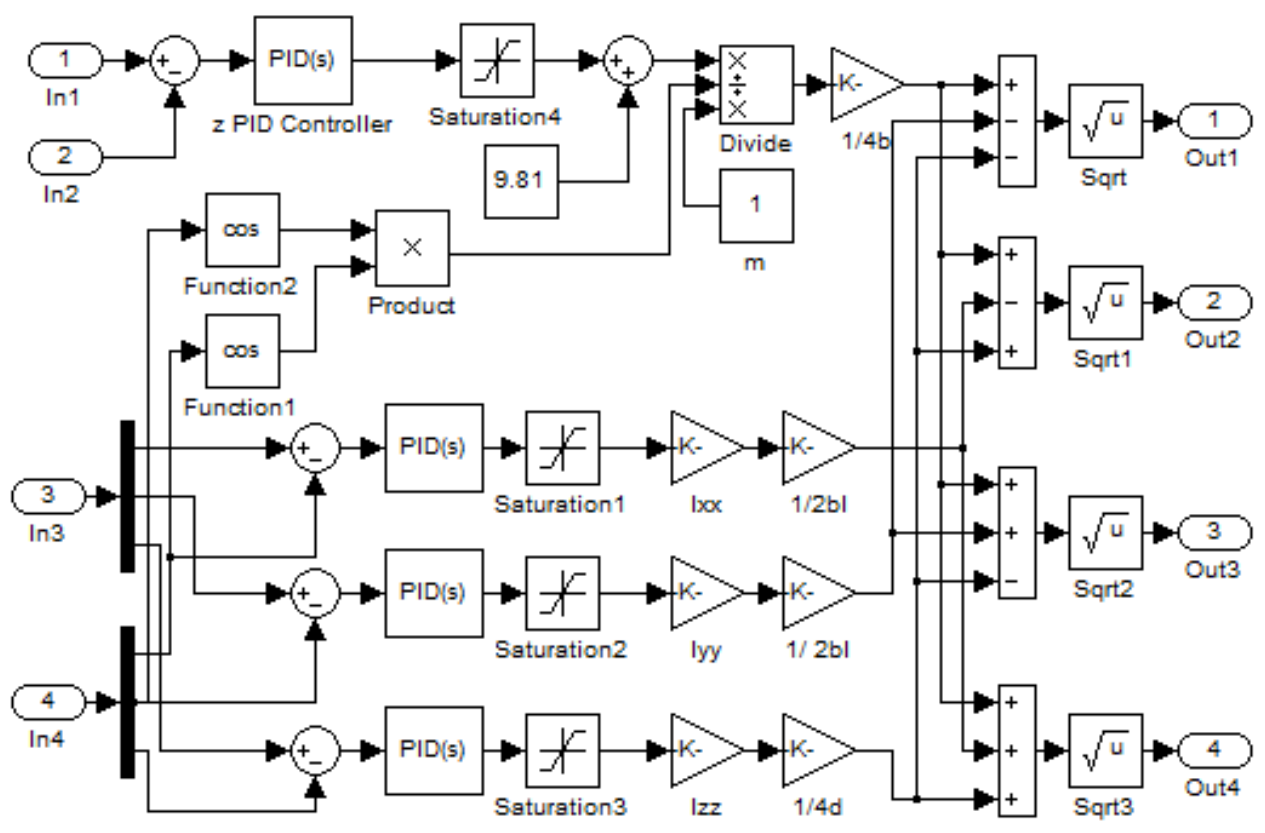

Figure 5. The internal structure of control block

In Figure 5, the input $1(\operatorname{In} 1)$ is the desired value of the height coordinate $(z)$, the input $2(\operatorname{In} 2)$ is the real value of the height coordinate which is measured by the height sensor. The role of the $z$ PID controller is controlling the height coordinate $(\mathrm{z})$, with input value is the error between In1 and In2. The input 3 (In3) is the desired value of the angle coordinate (roll $(\phi)$, pitch $(\theta)$ and yaw $(\psi)$ ). The input $4(\operatorname{In} 4)$ is the real value of the angle coordinate which is measured by the angle sensor. There are three angle controllers which are the PID controllers for the roll $(\phi)$, pitch $(\theta)$ and yaw $(\psi)$ angle. The output signals of the control block $\left(U_{1}, U_{2}, U_{3}, U_{4}\right)$ are through the inverse transform block to convert into the control signals as $\Omega_{1}, \Omega_{2}, \Omega_{3}$ and $\Omega_{4}$. The inverse transform block is built according to the equation (6). Figure 6 shows the Quad-rotor dynamic block which is built according to the equation (1).

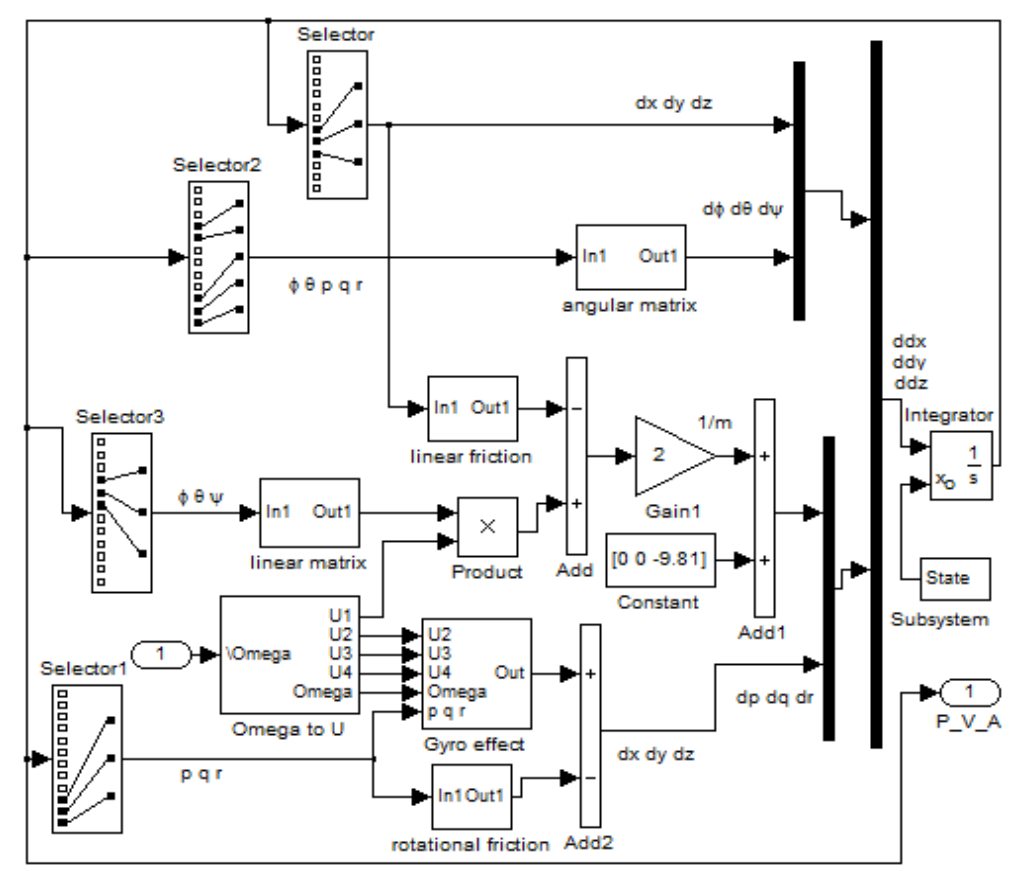

Figure 6. The Quad-rotor dynamic block 


\subsection{The model of double loop control structure}

The structure of single loop control has only four control signals, so Quad-rotor MAV only can be reached the height and stability. However, it is important to note that we have to well-known the orbit of the Quad-rotor MAV flight, and in order that Quad-rotor MAV can fly point-to-point, six coordinate variables must be controlled. So the double loop control structure system is proposed to do that.

The control structure with the double loop is presented as Figure 7, the internal function blocks in this control structure are built according to the equations (1)-(6).

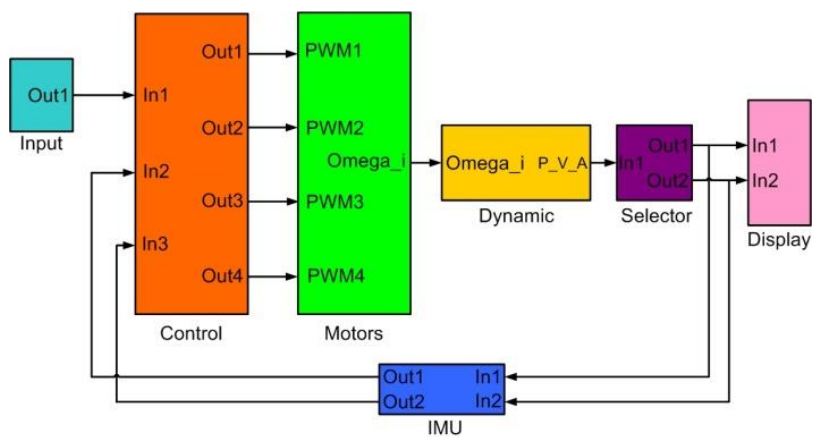

Figure 7. The double loop control structure

There are similarities between the double loop structure control and the single loop structure control relatively. However, there are some differences as: the input block in the double loop structure includes six desired values, which are the $x, y, z$ position coordinates and the Roll $(\phi)$, Pitch $(\theta)$, Yaw $(\psi)$ angle coordinates of the Quad-rotor. The selector block is used to select signals from the dynamic block into the IMU block, those selection signals after crossing IMU is the input of the control block. The difference of the selector block in the double loop control structure is that there are 6 signals $(x, y, z$ position coordinates and Roll $(\phi)$, Pitch $(\theta)$, Yaw $(\psi)$ angle coordinates) instead of 4 signals in the single loop control structure. The role of the display block is displaying the response of position coordinates $(x, y, z)$ and angles coordinates $(\phi, \theta, \psi)$ of the control system.

The control block is shown in detail as Figure 8. The role of the position control block is controlling the $x, y, z$ coordinates, with the input signals are In 1 and In $2 . \operatorname{In} 1$ is the desired values of $x, y, z$ coordinates and In 2 are the real values of $x, y, z$ coordinates from the sensor. The role of the transition block is calculating the angles coordinates $(\phi, \theta, \psi)$. The role of the attitude control block is controlling the angles coordinates, with In1 is the desired value of angles coordinates $(\phi, \theta, \psi)$ from the transition block, In2 is the real values of angle coordinates from the sensor. The role of the inversion block is converting the output values of the control block into the rotor speed value of four rotors $\Omega_{1}, \Omega_{2}, \Omega_{3}, \Omega_{4}$

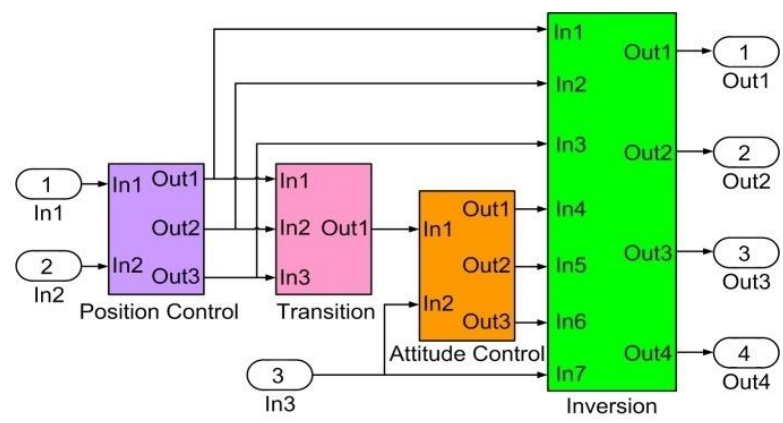

Figure 8. Control blocks in the double loop control structure

\section{THE SIMULATION RESULTS AND ANALYSIS}

We execute the simulation in two cases. The first case is the single loop structure control, the control signals are the height $(z)$, roll $(\phi)$, pitch $(\theta)$ and yaw $(\psi)$ coordinates. The second case is the double 
loop structure control, the control signals are the $x, y z$ position coordinates and the Roll $(\phi)$, Pitch $(\theta)$, Yaw $(\psi)$ angle coordinates.

\subsection{In the case of single loop structure}

After many experimental studies, the parameter values of the PID controller for the height (z), roll $(\phi)$, pitch $(\theta)$ and yaw $(\psi)$ coordinates are shown in Table 1 . Then, we test the ability of Quad-rotor to fly in space with the initial angles $(\phi, \theta, \psi)$ is $(0.2,0.2,0.5) \mathrm{rad}$. The simulation results are shown in Figure 9.

Table 1. The single loop control structure parameters

\begin{tabular}{cccc}
\hline & $\mathrm{Kp}$ & $\mathrm{Ki}$ & $\mathrm{Kd}$ \\
\hline Height & 24.1 & 5.9 & 20.2 \\
Roll & 0.80 & 0 & 0.20 \\
Pitch & 0.80 & 0 & 0.20 \\
Yaw & 0.39 & 0.19 & 0.19 \\
\hline
\end{tabular}
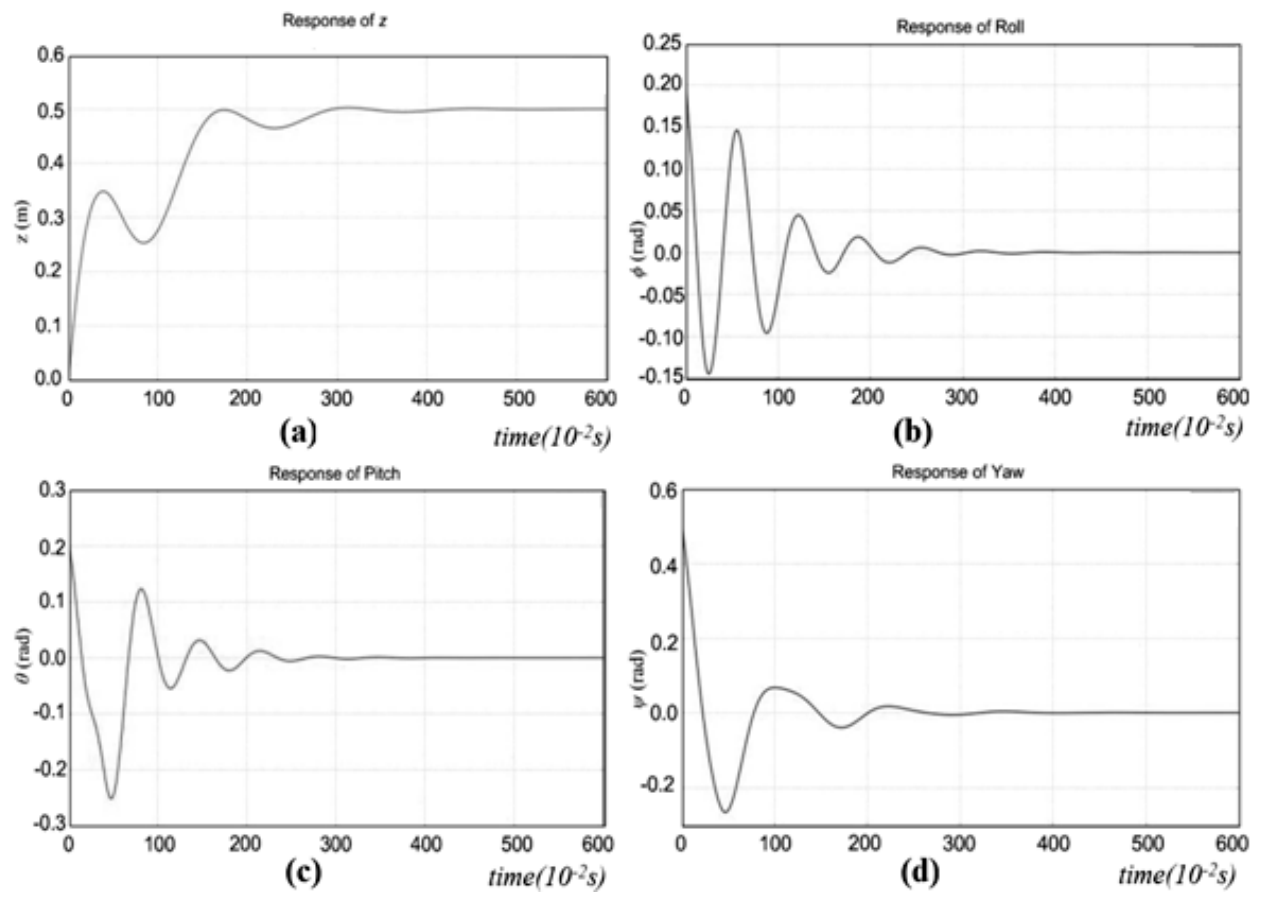

Figure 9. The simulation results of the single loop control structure: (a) Response of $z$; (b) Response of Roll; (c) Response of Pitch; (d) Response of Yaw

The simulation results show that the initial angles are not balanced, after controlling (after the 3rd second), the angle coordinates $(\phi, \theta, \psi)$ are balanced with the values $(0,0,0)$. The height coordinate $(z)$ reaches equilibrium at the height of $0.5 \mathrm{~m}$. After the transition time, all coordinates have good equilibrium, it is confirmed that the control structure works well and efficiently.

\subsection{In the case of double loop structure}

In the double loop structure control, we set the parameter values of the PID controller for the position coordinates $(x, y, z)$ and roll $(\phi)$, pitch $(\theta)$, yaw $(\psi)$ coordinates as in Table 2. Running the Quad-rotor fly from the position coordinates $(x, y, z)=(0,0,0)$ to $(0.1,0.1,1) \mathrm{m}$, and the angular coordinates $(\phi, \theta, \psi)$ change the desired value from $(0,0,0)$ to $(0.1,0.1,1)$ rad, the simulation results are shown in the Figure 10. 
Table 2. The double loop control structure parameters

\begin{tabular}{cccc}
\hline & $\mathrm{Kp}$ & $\mathrm{Ki}$ & $\mathrm{Kd}$ \\
\hline $\mathrm{X}$ & 0.078 & 0.0009 & 0.39 \\
$\mathrm{Y}$ & 0.078 & 0.0009 & 0.39 \\
$\mathrm{Z}$ & 24.1 & 5.9 & 20.1 \\
Roll & 0.69 & 0.0 & 0.13 \\
Pitch & 0.69 & 0.0 & 0.13 \\
Yaw & 0.059 & 0.009 & 0.19 \\
\hline
\end{tabular}
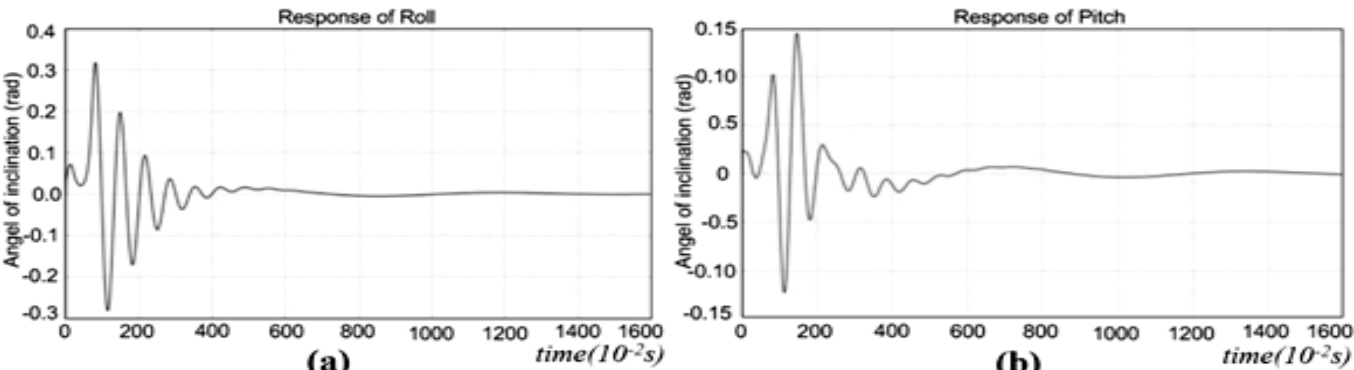

(a)
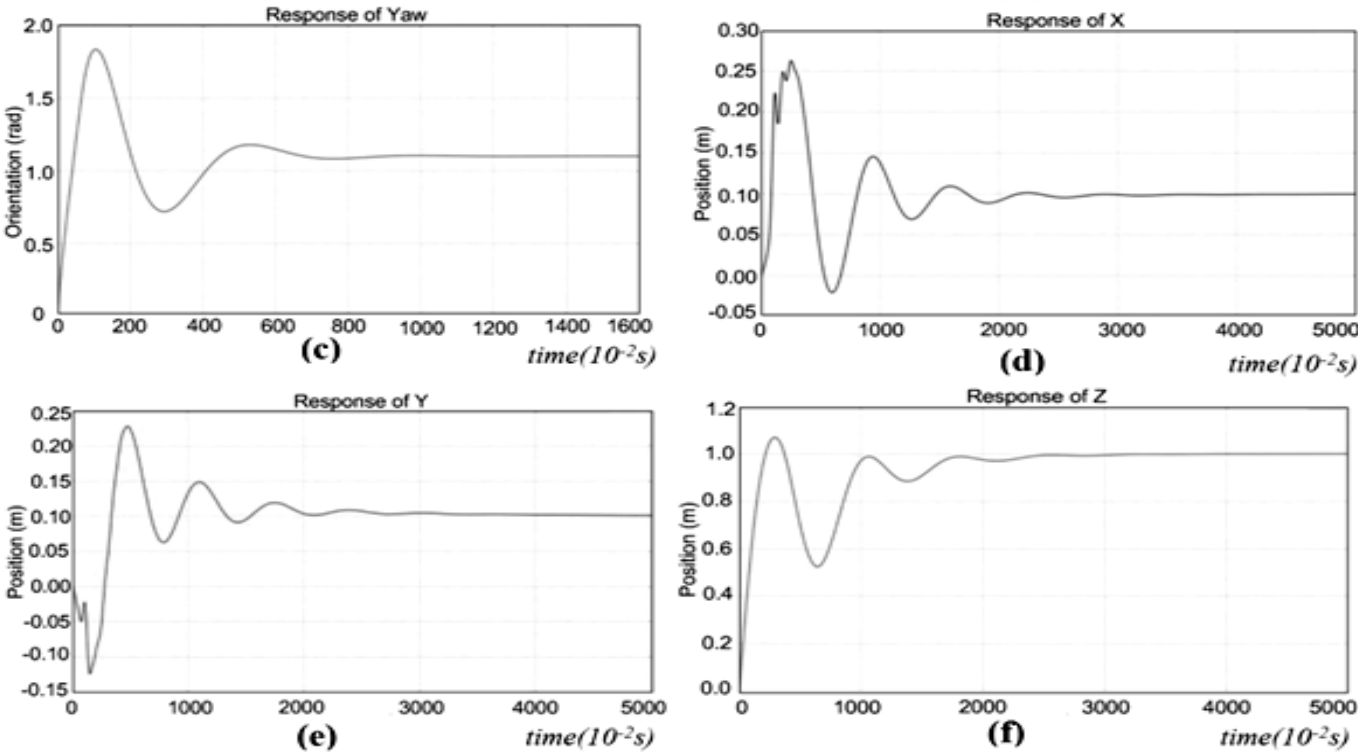

Figure 10. The simulation results of double loop control structure: (a) Response of Roll;

(b) Response of Pitch; (c) Response of Yaw; (d) Response of $x$; (e) Response of $y$; (f) Response of $z$

The simulation results in Figure 10 show that the roll $(\phi)$, pitch $(\theta)$, yaw $(\psi)$ coordinates attain the desired coordinates after about 4 seconds, the position coordinates $(x, y, z)$ attain the desired coordinates after about 10 seconds. After the transition time, all coordinates are stable, it is confirmed that the double loop control structure can control the Quad-rotor fly point-to-point well and efficiently.

\section{THE EXPERIMENTAL RESULTS}

After constructing the control structure for Quad-rotor and defining the control parameters, we install on the real device, the Quad-rotor MAV model is shown as Figure 11. 


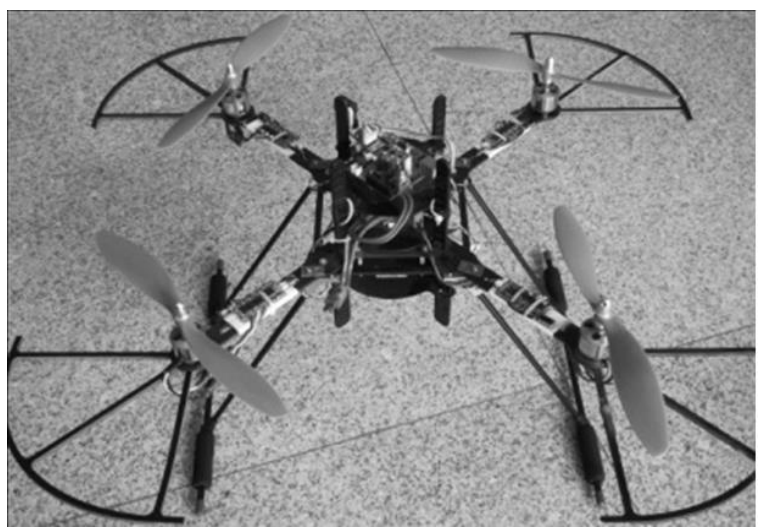

Figure 11. The Quad-rotor MAV model

Testing the system in the laboratory with the environment no disturbances caused by the wind. We control the angular coordinates $(\phi, \theta, \psi)$ changing the value from $(-38,-20,-68)$ to $(0,0,-40)$ degree, and the $x$, $y$ and $z$ coordinates changing the value from $(0.2,0.4,0.4)$ to $(0.8,0.6, .07) \mathrm{m}$, the experiment results are shown in Figure 12, which includes the real signal values of Roll, Pitch, Yaw angle coordinates and the $x, y, z$ position coordinates.
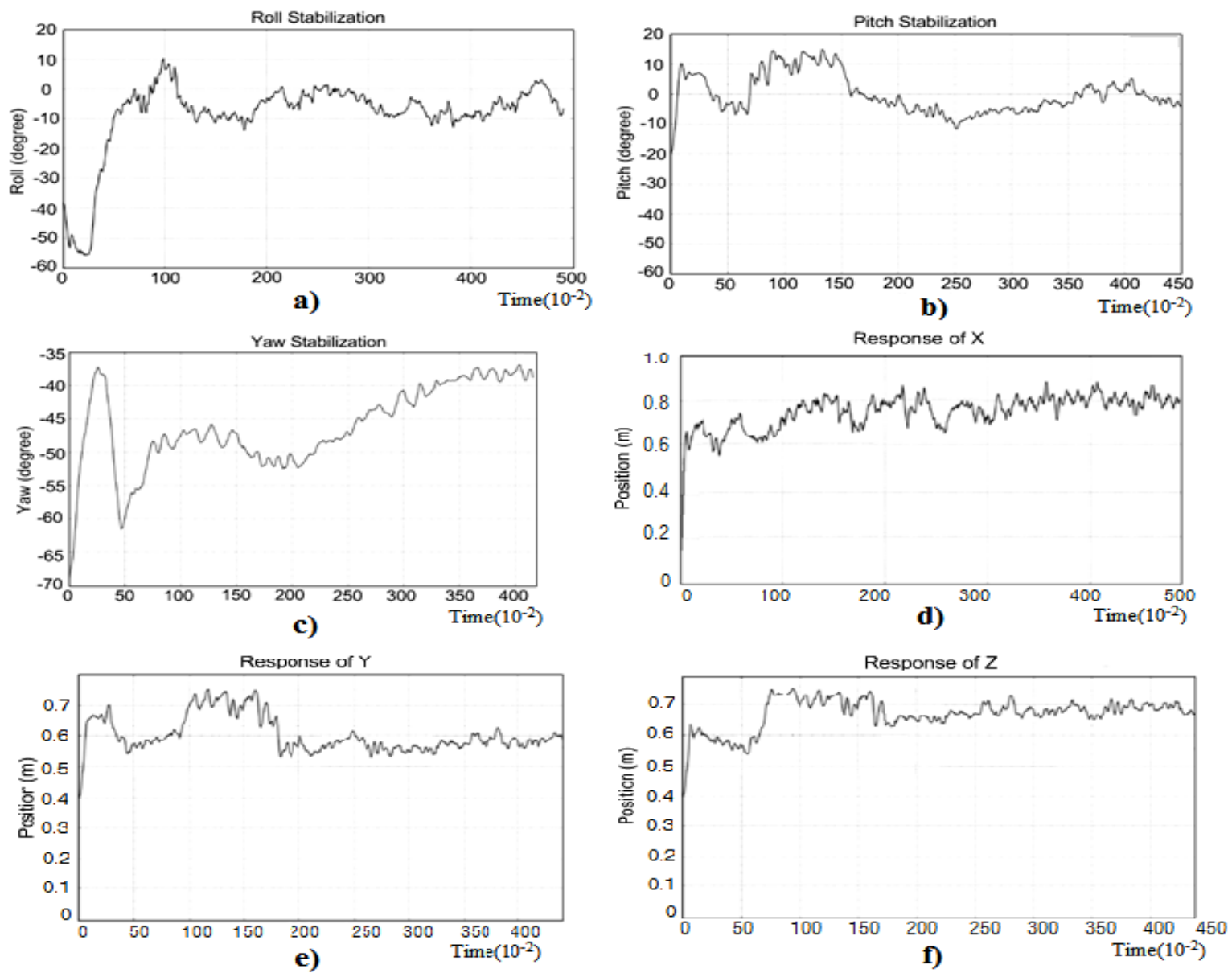

Figure 12. The experiment results: (a) Roll Stabilization; (b) Pitch Stabilization; (c) Yaw Stabilization; (d) Response of $x$; (e) Response of y; (f) Response of $\mathrm{z}$ 
The experiment results show that the responses of Roll, Pitch, Yaw coordinates $(\phi, \theta, \psi)$ and the $x, y$ and $z$ coordinates are good, all coordinates attain the desired value after about 4 seconds. However, the angular coordinates have the fluctuation about \pm 5 degrees. The amplitudes of this fluctuation are small and acceptable. So it is confirmed that the system control worked well and efficiently.

\section{CONCLUSION}

In this research, the authors have proposed two control structures for the Quad-rotor, particularly in the control system structure with the double loop. The simulation results are presented to prove that six coordinates $(x, y, z, \phi, \theta, \psi)$ can be controlled by four rotors, so the Quad-rotor can fly point-to-point exactly in the space. Then, the experimental results make certain that the Quad-rotor can go to the desired position and the desired direction with the short transition time, so the proposed control structure can be applied in practice with the low cost and the high-efficiency.

\section{REFERENCES}

[1] M. S. M. Ashraf, et al., "Modelling and Parameters Identification of a Quadrotor Using a Custom Test Rig," International Journal of Power Electronics and Drive Systems (IJPEDS), vol/issue: 9(2), pp. 865-872, 2018.

[2] A. H. Ginting, et al., "Attitude Control of Quadrotor using PD Plus Feedforward controller on SO (3)," International Journal of Electrical and Computer Engineering (IJECE), vol/issue: 8(1), pp. 566-575, 2018.

[3] M. R. Eressa, et al., "PID and neural net controller performance comparsion in MAV pitch attitude control," IEEE International Conference on Systems, Man, and Cybernetics (SMC), pp. 000762 - 000767, 2016.

[4] D. Zhang, et al., "Adaptive dual fuzzy PID control method for longitudinal attitude control of tail-sitter MAV," 22nd International Conference on Automation and Computing (ICAC), pp. 378 - 382, 2016.

[5] R. R. Benrezki, et al., "Passive fault tolerant control of Quadrotor MAV using a nonlinear PID," IEEE International Conference on Robotics and Biomimetics (ROBIO), pp. 1285 - 1290, 2015.

[6] Shuai A., et al., "Self-tuning of PID controllers design by adaptive interaction for Quadrotor MAV," IEEE Chinese Guidance, Navigation and Control Conference (CGNCC), pp. 1547 - 1552, 2016.

[7] S. Bagheri, et al., "Beneficially combining LQR and PID to control longitudinal dynamics of a SmartFly MAV," IEEE 7th Annual Information Technology, Electronics and Mobile Communication Conference (IEMCON), pp. 1 6, 2016.

[8] S. G. V'azquez and J. M. Valenzuela, "A New Nonlinear PI-PID Controller for Quadrotor Posture Regulation," Electronics, Robotics and Automotive Mechanics Conference, pp. 642-647, 2010.

[9] Weiyong S., et al., "Fuzzy PID controller for MAV tracking moving target," 29th Chinese Control And Decision Conference (CCDC), pp. 3023 - 3027, 2017.

[10] Y. Mou, et al., "The flight control of micro quad-rotor MAV based on PID," 31st Youth Academic Annual Conference of Chinese Association of Automation (YAC), pp. 353 - 356, 2016.

[11] J. M. Selfridge and G. Tao, "A multivariable adaptive controller for a Quadrotor with guaranteed matching conditions," American Control Conference (ACC), pp 26-31, 2014.

[12] W. Saengphet, et al., "Implementation of system identification and flight control system for MAV," IEEE International Conference on Robotics and Automation, pp. 678 - 683, 2017.

[13] N. M. Raharja, et al., "Hovering Control of Quadrotor Based on Fuzzy Logic," International Journal of Power Electronics and Drive Systems (IJPEDS), vol/issue: 8(1), pp. 492-504, 2017.

[14] F. Şenkul and E. Altuğ, "Adaptive control of a tilt - roll rotor Quadrotor MAV," International Conference on Miniature Aircraft Systems (ICUAS), pp. 1132-1137, 2014.

[15] M. Żugaj, et al., "Thrust pulse control for MAV control system reconfiguration," 21st International Conference on Methods and Models in Automation and Robotics (MMAR), pp. 708 - 713, 2016.

[16] O. C. Carholt, et al., "Design, modelling and control of a Single Rotor MAV," 24th Mediterranean Conference on Control and Automation (MED), pp. 840 - 845, 2016.

[17] Y. Wang, et al., "Fuzzy radial basis function neural network PID control system for a Quadrotor MAV based on particle swarm optimization," IEEE International Conference on Information and Automation, pp. 2580 - 2585, 2015.

[18] C. R. Ashokkumar, et al., "Time response bounds in nonlinear MAV control," International Conference on Miniature Aircraft Systems (ICUAS), pp. 16 - 22, 2017.

[19] J. Ordaz, et al., "Quad rotor-MAV stabilization by predictor based control," International Conference on Miniature Aircraft Systems (ICUAS), pp. 29 - 35, 2017.

[20] O. Araar, et al., "Identification \& control of a multirotor MAV in the presence of actuator asymmetry," 25th Mediterranean Conference on Control and Automation (MED), pp. 1035 - 1040, 2017.

[21] M. N. Duc, et al., "The Quadrotor MAV System using PID Control," IEEE International Conference on Mechatronics and Automation (ICMA), pp. 506-510, 2015.

[22] M. N. Duc and T. N. Trong, "The Quadrotor MAV System using double-loop PID Control," International Journal of Advanced and Applied Sciences, pp. 57-61, 2016. 